\title{
Aspectos associados com a qualidade de vida de pessoas que sofreram queimaduras
}

\section{Aspects associated with the quality of life of people who suffered burns}

\author{
Uiara Aline de Oliveira Kaizer' $\bullet$ Laila Silva Divino ${ }^{2} \bullet K^{2}$ erolayne Feliciano Lemes ${ }^{3}$ \\ Sthefany Scarlet Patricio Bertoldo ${ }^{4}$ Elaine Aparecida Rocha Domingues ${ }^{5}$ Natalia Gonçalves $^{6}$
}

\begin{abstract}
RESUMO
Objetivo: relacionar os dados sociodemográficos e clínicos com a qualidade de vida de pessoas que sofreram queimaduras. Método: estudo descritivo e transversal, realizado em centro especializado de novembro de 2013 a fevereiro de 2014. Foram entrevistados 100 adultos, com queimaduras de grau II e/ou III, utilizando-se de escalas sobre qualidade de vida. Resultados: Houve correlação significativa entre a superfície corporal queimada e os domínios Físico $(r=-0,2683)$, Sensibilidade da pele $(r=-0,2 \mid 27)$, Tratamento $(r=0,2 \mid 24)$, Relações Interpessoais $(r=-0,2014)$ e escore total da escala de estado de saúde $(r=-0,247 \mid)$. Quanto ao tempo de tratamento, observaram-se associações com os domínios Afeto e Imagem Corporal ( $r=-$ $0,2159)$, Habilidades para funções simples $(r=0,4220)$ e Relações Interpessoais $(r=-0,2917)$. Houve diferenças no domínio Relações Interpessoais para o sexo feminino. Conclusão: Mulheres perceberam pior sua qualidade de vida em termo das relações pessoais e, o tempo de tratamento e a extensão da queimadura afetaram negativamente o estado de saúde. Descritores: Qualidade de vida; Queimaduras; Enfermagem; Medidas de resultados relatados pelo paciente;Avaliação de resultados em cuidados de saúde.
\end{abstract}

\begin{abstract}
Objective: to verify the association between sociodemographic and clinical data with the quality of life of people who suffered burns. Method: a descriptive and transversal study, carried out in a specialized center from November 2013 to February 2014. 100 adults were interviewed, with burns of grade II and / or III, using scales on quality of life. Results: There was a significant correlation between the burned body surface and the Physical $(r=-0.2683)$, Skin Sensitivity $(r=-0.2127)$, Treatment $(r=$ $0.2124)$, Interpersonal Relations $(r=-0,2014)$ and total score of the health status scale $(r=-0.2471)$. As for the treatment time, associations were observed with the domains Affect and Body Image $(r=-0.2159)$, Skills for simple functions $(r=$ $0.4220)$ and Interpersonal Relations $(r=-0.2917)$. There were differences in the Interpersonal Relations domain for females. Conclusion:Women perceived their quality of life worse in terms of personal relationships and the length of treatment and the extent of the burn negatively affected their health status.
\end{abstract}

Keywords: Quality of life; Burns; Nursing, Patient Reported Outcome Measures; Outcome Assessment, Health Care

I Enfermeira, Doutora e Mestre em Ciências da Saúde pela Unicamp. Estomaterapeuta TiSobest. Responsável técnica pelo ambulatório de Feridas da Prefeitura de Sorocaba- SP. Contribuição: Concepção e projeto, análise, interpretação dos dados, e participação ativa na discussão dos resultados; Redação do artigo; Revisão e aprovação final de versão a ser publicada; Concordância com todos os aspectos do manuscrito em termos de veracidade ou integridade das informações.

2 Estudante de Enfermagem da Universidade Vale do Rio Verde, Três Corações, MG. Contribuição: Interpretação dos dados, e participação ativa na discussão dos resultados; Redação do artigo; Revisão e aprovação final de versão a ser publicada; Concordância com todos os aspectos do manuscrito em termos de veracidade ou integridade das informações.

3 Estudante de Enfermagem da Universidade Vale do Rio Verde, Três Corações, MG. Contribuição: Interpretação dos dados, e participação ativa na discussão dos resultados; Redação do artigo; Revisão e aprovação final de versão a ser publicada; Concordância com todos os aspectos do manuscrito em termos de veracidade ou integridade das informações.

4 Estudante de Enfermagem da Universidade Vale do Rio Verde, Três Corações, MG. Contribuição: Interpretação dos dados, e participação ativa na discussão dos resultados; Redação do artigo; Revisão e aprovação final de versão a ser publicada; Concordância com todos os aspectos do manuscrito em termos de veracidade ou integridade das informações.

5 Enfermeira, Doutora em Ciências da Saúde. Docente da Universidade Vale do Rio Verde, Três Corações, MG. Contribuição Análise, interpretação dos dados, e participação ativa na discussão dos resultados; Redação do artigo e revisão crítica relevante do conteúdo intelectual; Revisão e aprovação final de versão a ser publicada; Concordância com todos os aspectos do manuscrito em termos de veracidade ou integridade das informações.

6 Enfermeira. PhD Professora Adjunta do Departamento de Enfermagem e do Programa de Pós-graduação em Enfermagem da Universidade Federal de Santa Catarina. Contribuição: Interpretação dos dados, e participação ativa na discussão dos resultados; Redação do artigo e revisão crítica relevante do conteúdo intelectual; Revisão e aprovação final de versão a ser publicada; Concordância com todos os aspectos do manuscrito em termos de veracidade ou integridade das informações.

Autor Correspondente: Uiara Aline de Oliveira Kaizer - Endereço:Tessália Vieira de Camargo, I26- Cidade Universitária,Campinas - Orcid: https://orcid.org/00000002-9| I5-8043 - tel: +55 15 997| 238336 - uiara_oliveira@hotmail.com 


\section{INTRODUÇÃO}

A queimadura compromete a integridade funcional da pele, responsável pela homeostase hidroeletrolítica, controle da temperatura interna, flexibilidade e lubrificação da superfície corporal. É uma lesão dos tecidos orgânicos em decorrência de um trauma de origem térmica, que varia desde uma pequena boIha até formas mais graves, com desfiguração, incapacidade e óbito. Além da gravidade, geram alterações psicológicas e sociais, impactando diretamente na qualidade de vida (QV) dos indivíduos ${ }^{(1)}$, devido a distintos fatores, dentre eles, a extrema dor durante o processo de tratamento ${ }^{(2)}$.

Com os avanços no tratamento de queimados, houve uma redução na mortalidade na fase hospitalar, na qual o paciente pode apresentar disfunção de múltiplos órgãos e infecções sistêmicas. Contudo, com o aumento da sobrevivência, há um grande número de pessoas com desfigurações, amputações, perda de função e esses aspectos podem levar a alterações nas atividades cotidianas, nas representações sociais e na percepção de autoimagem dessas pessoas, aspectos que também impactam a $\mathrm{QV}{ }^{(3)}$.

Um dos principais desafios da equipe de saúde é a reabilitação e reintegração dos pacientes na sociedade, visto que além de apresentar sequelas estéticas e funcionais, os aspectos psicológicos também podem estar. A vivência entre os profissionais durante o período de tratamento é de extrema importância para que se chegue ao resultado esperado. $O$ paciente deve ser sempre o centro do cuidado e cada um da equipe de saúde deve atuar de forma singular, e estar unidos em prol do bem-estar físico e mental do indivíduo, aumentando assim os índices de recuperação e a meIhora da percepção da QV ${ }^{(4)}$.

A QV é a percepção do indivíduo de sua inserção na vida, no contexto da cultura e sistemas de valores nos quais ele vive e em relação aos seus objetivos, expectativas, padrões e preocupações. Envolve o bem-estar espiritual, físico, mental, psicológico e emocional, além de relacionamentos sociais, como família e amigos e, também, saúde, educação, habitação saneamento básico e outras circunstâncias da vida ${ }^{(5)}$.

Logo, emergiram as seguintes questões problemas: como está a qualidade de vida de pacientes que vítimas de queimadura? Quais aspectos sociais e clínicos que interferem na qualidade de vida? Assim, o objetivo do estudo é relacionar os dados sociodemográficos e clínicos com a qualidade de vida de pessoas que sofreram queimaduras.

A relevância do estudo está diretamente relacionada a avaliação da $\mathrm{QV}$, pois é de suma importância que $\circ$ tratamento das queimaduras seja $\circ$ menos doloroso e, mais eficaz possível, com o intuito de diminuir o impacto causado pelo trauma e que o paciente recupera o máximo de suas funções. Quando se finaliza $\circ$ tratamento hospitalar, deve- se compreender que o mesmo perpassa a cura da ferida, e deve ser acrescido ao tratamento psicológico e um preparo para o enfrentamento das dificuldades em meio à sociedade ${ }^{(6)}$. Ademais, identificar quais os fatores interferem de maneira negativa na QV norteia o profissional a prestar assistência integral ao paciente vítima de queimaduras, abarcando todas as dimensões durante o processo de tratamento e reabilitação.

\section{METÓdO}

Trata-se de estudo com abordagem quantitativa, do tipo analítico de dados secundários referentes à pesquisa de mestrado ${ }^{(7)}$ sobre as propriedades psicométricas do "Freiburg Life Quality Assessment Wound" em pacientes que sofreram queimaduras. $O$ estudo foi conduzido em um Centro de Tratamento de Queimados de um Hospital Estadual, localizado em uma cidade do interior do estado de São Paulo, o qual é referência em atendimento de nível terciário na região sudoeste do estado.

A amostra, por conveniência, foi composta de pessoas que sofreram queimaduras que frequentavam o serviço, em seguimento ambulatorial e internação no Centro de Tratamento de Queimados. A coleta de dados ocorreu entre os meses de novembro de 2013 a fevereiro de 20I4. Para tal, foram seguidos, os seguintes critérios de inclusão: queimadura de grau II e/ou III (de acordo com a classificação no prontuário), com idade igual ou superior a 18 anos e que estavam aptos a responder o questionário; e de exclusão: pessoas com queimadura de grau I e que apresentaram qualquer dificuldade de comunicação verbal que os impedia de responder à entrevista. Assim, a amostra foi composta de 100 sujeitos, sendo que somente três pacientes se recusaram a participar do estudo, dois por motivo de "pressa" no atendimento (SIC) e uma usuária de entorpecentes que estava na internação.

Os dados foram coletados pela pesquisadora por meio de entrevista, utilizando-se de três questionários, a saber, instrumento sobre dados sociodemográfico e clínicos, Burn Specific Health Scale - Revisado(BSHS-R) e World Health Organization Quality of Life instrument- Bref (WHOQOL-abreviado).

Para caracterização dos participantes, foi adaptado questionário desenvolvido previamente ${ }^{(8)}$ e consideradas as seguintes variáveis: data de nascimento, sexo, estado civil e escolaridade, porcentagem da área com queima- 
duras, agente etiológico, locais do corpo queimados, profundidade e motivo da queimadura.

O estado de saúde foi avaliado pela Burn Specific Health Scale-Revised (BSHS-R), elaborado em 1994(9), sendo específica para avaliar este construto em pessoas que sofreram queimaduras. Foi traduzida e adaptada para a língua portuguesa e validada para o Brasil em 2008(8). É composta por trinta e um itens distribuídos em seis domínios: Afeto e Imagem Corporal (oito itens), Sensibilidade da Pele (cinco itens), Habilidades para funções simples (quatro itens), Tratamento (cinco itens), Trabalho (quatro itens) e Relações Interpessoais (cinco itens) ${ }^{(8)}$. Os valores da BSHS-R podem variar de um a cinco pontos e o intervalo possível de $3 \mathrm{I}$ a $\mid 55$, sendo que maiores pontuações indicam melhor estado de saúde. No estudo de validação para o Brasil, a BSHS-R apresentou alfa de Cronbach de 0,94 para a escala total, com variação de 0,74 a 0,94 para os domínios ${ }^{(8)}$.

A QV foi avaliada pelo instrumento WHOQOL -abreviado, desenvolvido em $1998^{(10)}$, traduzido para a língua portuguesa e validado para o Brasil em 2000(11). Composto por vinte e seis itens, sendo que dois deles referem-se à temática geral: $\mathrm{QV}$ e saúde e os demais itens representam cada uma das facetas que compõe o instrumento original (WHOQoL-100), em que cada faceta é avaliada por apenas uma questão. Os itens são divididos em quatro domínios: Físico, Psicológico, Relações Sociais e Meio-Ambiente. As questões do WHOQOL-abreviado possuem quatro tipos de escalas de respostas: Intensidade, Capacidade, Frequência e Avaliação, graduadas por uma escala com pontuações do tipo Likert que varia de um a cinco, em que um é o extremo negativo ( $0 \%$ ) e o cinco o extremo positivo (100\%). As vinte e seis questões possuem escores que variam de $0 \%$ a $100 \%$, em que os menores escores correspondem à pior $\mathrm{QV}$. $\mathrm{O}$ instrumento não admite escore total de $\mathrm{QV}$, sendo cada domínio pontuado de forma independente. A confiabilidade da versão WHOQOL-abreviado para o Brasil foi avaliada por meio da consistência interna, com coeficiente alfa Cronbach de $0,90^{(11)}$. A escolha por esses instrumentos foi devida às suas propriedades de medida satisfatórias, como validade e confiabilidade, já terem sido mostradas anteriormente ${ }^{(8,11)}$.

Os dados foram digitados no programa Microsoft Office Excel 2007 e posteriormente transportados para o software estatístico SAS versão 9.2. Os dados de caracterização sociodemograficas e clínica, e o escore total dos instrumentos de QV foram apresentados por meio de estatísticas descritivas. As correlações entre as variáveis quantitativas foram avaliadas por meio do coeficiente de correlação de Spearman. Para avaliação dos valores foi utilizado o referencial de Cohen (1988) que sugere a seguinte classificação do coeficiente de correlação: 0,1 a 0,29 (fraca), 0,30 a 0,49 (moderada) e maior ou igual a 0,50 (forte) ${ }^{(12)}$. Para as comparações envolvendo uma variável qualitativa e uma variável quantitativa foi aplicado o teste $t$ de Student não pareado ou o teste não-paramétrico de Mann-Whitney, de acordo com a distribuição dos dados. Para todas as análises foi considerado um nível de significância menor ou igual a $5 \%$. O estudo seguiu as recomendações da Resolução 466/2012 do ConseIho Nacional de Saúde e foi aprovado pelo Comitê de Ética em Pesquisa da Faculdade de Ciências Médicas da Unicamp, tendo sido aprovado sob o Parecer $n^{\circ}$ $338.354 / 2013$.

\section{RESULTADOS}

Foram entrevistados no total de 100 pessoas que sofreram queimaduras. Desses, $59 \%$ eram do sexo masculino, solteiros (47\%) e $45 \%$ cursaram o ensino médio. A média de idade foi de 39 anos (desvio padrão (D.P.) $=14,9$; 18-79) A maioria dos acidentes aconteceu em ambiente doméstico $(n=56)$, seguido do acidente no ambiente de trabalho $(n=40)$ e tentativa de autoextermínio $(n=4)$. Em relação aos dados clínicos, o agente etiológico da queimadura mais prevalente foi a escaldadura (47\%), atingindo mais que uma parte do corpo (4l\%), com profundidade classificada em segundo grau (7I\%), e a maioria dos entrevistados informou a que apresentava sequela do tipo estética (80\%) (Tabela I). A porcentagem de superfície corporal média foi de 7,2 (D.P.=10,8; mínimo $0,1$ e máximo 60,0$)$. O tempo médio de tratamento para a queimadura foi de 67,12 meses (D.P. $=\mid 46,55$; mínimo I e máximo 780).

Em relação à qualidade de vida, os participantes apresentam melhor escore para o domínio Psicológico (média=72, I, D.P.= 12,0) e para o estado de saúde, nos domínios Afeto e imagem corporal (média=30,9. D.P. $=7,3$ ) e Relações Interpessoais (média= 22,8, D.P.= 3,6) (Tabela 2).

Constatou-se associação significativa e fraca entre SCQ e os domínios Físico do WHOQOL- abreviado $(r=-0,268)$, Tratamento $(r=-0,2 \mid 2)$ e Relações Interpessoais $(r=-0,20 I)$ da BSHS-R. Ainda, houve correlação de magnitude fraca entre tempo de tratamento das queimaduras e os domínios Afeto e Imagem corporal $(r=-0,215)$ e Relações Interpessoais $(r=-0,291)$, e moderada com o domínio Habilidades para funções simples $(r=0,422)$ (Tabele 3).

Quando comparado os domínios das escalas com a categoria sexo, houve diferença estatisticamente significante apenas nas Relações Interpessoais (Tabela 4) 
TABELA 1 - Características pessoais e clínicas de pessoas que sofreram queimaduras. Campinas, SP, Brasil, 2014

\begin{tabular}{|c|c|c|}
\hline Variável & $\mathrm{n}$ & $\%$ \\
\hline \multicolumn{3}{|l|}{ Sexo } \\
\hline Feminino & 41 & 41,0 \\
\hline Masculino & 59 & 59,0 \\
\hline \multicolumn{3}{|l|}{ Estado civil } \\
\hline Solteiro & 47 & 47,0 \\
\hline Casado & 43 & 43,0 \\
\hline Divorciado & 6 & 6,0 \\
\hline Viúvo & 4 & 4,0 \\
\hline \multicolumn{3}{|l|}{ Escolaridade } \\
\hline Nenhum & 1 & 1,0 \\
\hline Fundamental incompleto & 44 & 44,0 \\
\hline Ensino médio & 45 & 45,0 \\
\hline Superior & 10 & 10,0 \\
\hline \multicolumn{3}{|l|}{ Agente etiológico } \\
\hline Escaldo & 47 & 47,0 \\
\hline Contato & 14 & 14,0 \\
\hline Fogo & 31 & 31,0 \\
\hline Radiação & 2 & 2,0 \\
\hline Eletricidade & 6 & 6,0 \\
\hline \multicolumn{3}{|l|}{ Locais do corpo } \\
\hline MMSS* & 30 & 30,0 \\
\hline $\mathrm{MMII}^{* *}$ & 21 & 21,0 \\
\hline Cabeça e pescoço & 2 & 2,0 \\
\hline Tronco & 6 & 6,0 \\
\hline Mais que uma parte do corpo & 41 & 41,0 \\
\hline \multicolumn{3}{|l|}{ Grau } \\
\hline 2 & 71 & 71,0 \\
\hline $2-3$ & 20 & 20,0 \\
\hline 3 & 9 & 9,0 \\
\hline \multicolumn{3}{|l|}{ Circunstâncias do trauma } \\
\hline Autoextermínio & 4 & 4,0 \\
\hline Acidente Doméstico & 56 & 56,0 \\
\hline Acidente de trabalho & 40 & 40,0 \\
\hline \multicolumn{3}{|l|}{ Sequela perspectiva do paciente } \\
\hline Funcionais & 1 & 1,0 \\
\hline Estéticas & 80 & 80,0 \\
\hline Ambas & 19 & 19,0 \\
\hline
\end{tabular}

*MMSS: Membros Superiores. ${ }^{* *}$ MMII: Membros Inferiores. 
TABELA 2 - Qualidade de vida e estado de saúde pessoas que sofreram queimaduras ( $n=100)$, Campinas, SP, Brasil, 2014

\begin{tabular}{c|c|c|c|c|c}
\hline Domínios & Média & Desvio-padrão & Mínimo & Mediana & Máximo \\
\hline WHOQOL-Abreviado & & & & & \\
\hline Físico & 59,1 & 17,1 & 0,0 & 60,7 & 100,0 \\
\hline Psicológico & 72,2 & 12,0 & 20,8 & 75,0 & 95,8 \\
\hline Social & 69,7 & 14,9 & 25,0 & 75,0 & 100,0 \\
\hline Meio Ambiente & 60,6 & 12,4 & 25,0 & 62,5 & 96,9 \\
\hline BSHS-R & & & & & \\
\hline Afeto e Imagem corporal & 30,9 & 7,3 & 9,0 & 32,0 & 40,0 \\
\hline Sensibilidade da pele & 13,1 & 4,6 & 5,0 & 12,0 & 23,0 \\
\hline Habilidades para funções simples & 15,7 & 4,4 & 4,0 & 17,0 & 20,0 \\
\hline Trabalho & 10,7 & 5,4 & 4,0 & 9,0 & 20,0 \\
\hline Tratamento & 18,8 & 5,1 & 5,0 & 20,0 & 25,0 \\
\hline Relações interpessoais & 22,9 & 3,7 & 7,0 & 25,0 & 25,0 \\
\hline Escore Total & 112,1 & 19,7 & 47,0 & 113,5 & 151,0 \\
\hline
\end{tabular}

TABELA 3 - Associação entre os domínios do WHOQOL-abreviado e BSHS-R e idade, tempo de queimadura e superfície corporal queimada. Sorocaba, SP, Brasil 2014

\begin{tabular}{c|c|c|c}
\hline Domínios & Idade & $\begin{array}{c}\text { Tempo de tratamento das } \\
\text { queimaduras }\end{array}$ & SCQ \\
\hline WHOQOL-Abreviado & & & $-0,2683^{*}$ \\
\hline Físico & 0,0631 & $-0,0599$ & 0,0611 \\
\hline Psicológico & $-0,0401$ & $-0,0908$ & $-0,0352$ \\
\hline Social & 0,0892 & $-0,0866$ & 0,0295 \\
\hline Meio Ambiente & 0,0211 & $-0,1007$ & $-0,0798$ \\
\hline BSHS-R & & & $-0,2127^{*}$ \\
\hline Afeto e Imagem corporal & 0,0651 & $-0,2159^{*}$ & $-0,1728$ \\
\hline Sensibilidade da pele & 0,0462 & $-0,0432$ & $-0,1288$ \\
\hline Habilidades para funções simples & 0,1914 & $0,4220^{*}$ & $-0,2124^{*}$ \\
\hline Trabalho & 0,1149 & 0,0807 & $-0,2014^{*}$ \\
\hline Tratamento & 0,0755 & $-0,0055$ & $-0,2471^{*}$ \\
\hline Relações interpessoais & $-0,1171$ & $-0,2917^{*}$ & $-0,0076$ \\
\hline
\end{tabular}

Correlação de Spearman. *valor $p<0,05$.

TABELA 4 - Comparação entre sexo e os domínios das escalas WHOQOL-abreviado e BSHS-R. Sorocaba, SP, Brasil, 2014

\begin{tabular}{l|c|c|c}
\hline \multicolumn{1}{c|}{ Domínios } & \multicolumn{2}{c}{$\begin{array}{c}\text { Sexo } \\
\text { Média (D.P.) }\end{array}$} & \multicolumn{1}{c}{$\begin{array}{c}\text { Masculino } \\
(\mathrm{n}=59)\end{array}$} \\
\hline WHOQOL-Abreviado & $\begin{array}{c}\text { Feminino } \\
(\mathrm{n}=41)\end{array}$ & \\
\hline Físico & & $58,6(15,4)$ & $0,741^{*}$ \\
\hline Psicológico & $59,8(19,6)$ & $73,4(10,9)$ & $0,323^{* *}$ \\
\hline Social & $70,4(13,4)$ & $70,9(15,3)$ & $0,108^{* *}$ \\
\hline Meio Ambiente & $68,1(14,3)$ & $59,7(11,6)$ & $0,461^{* *}$ \\
\hline BSHS-R & $61,8(13,5)$ & & \\
\hline Afeto e Imagem corporal & & $31,8(6,4)$ & $0,249^{* *}$ \\
\hline Sensibilidade da pele & $29,6(8,3)$ & $13,5(4,9)$ & $0,468^{* *}$ \\
\hline Habilidades para funções simples & $12,7(4,2)$ & $15,7(4,2)$ & $0,570^{* *}$ \\
\hline Trabalho & $15,7(4,9)$ & $10,0(5,3)$ & $0,084^{* *}$ \\
\hline Tratamento & $11,7(5,5)$ & $19,1(4,6)$ & $0,701^{* *}$ \\
\hline Relações interpessoais & $18,4(5,7)$ & $23,5(3,2)$ & $0,031^{* *}$ \\
\hline Escore Total & $22,0(4,1)$ & $113,6(18,1)$ & $0,387^{*}$ \\
\hline
\end{tabular}

* $p$-valor obtido por meio do teste t de Student não pareado. ** p-valor obtido por meio do teste de Mann-Whitney. 


\section{DISCUSSÃO}

Este estudo objetivou verificar a relação entre dados sociodemográficos e clínicos com a qualidade de vida de pessoas que sofreram queimaduras. Este tema é de grande relevância social, pois o corpo é um objeto modelado pela sociedade, sendo impossível estabelecer uma distinção entre o que diz respeito à pessoa e aquilo que é colocado em padrão da sociedade. As queimaduras acarretam danos físicos nas habilidades e capacidade funcionais dos indivíduos para a realização de tarefas. Ainda, repercutem negativamente nos aspectos emocionais, com sentimentos de negação, medo, ansiedade, bem como comprometimentos da autonomia e da imagem corporal $^{(13)}$. Esses aspectos podem afetar a percepção da qualidade de vida da pessoa que sofreu queimaduras sendo inferior à da população em geral, refletido em múltiplas dimensões da vida.

$\mathrm{Na}$ literatura, autores ${ }^{(14)}$ recomendam aprofundar o corpo de conhecimento da enfermagem em relação à qualidade de vida, a fim de identificar as tendências estatísticas atuais, e fornecer evidências científicas quantitativas que informam a teoria e a criação de novas intervenções. Ainda, é necessário que se busque aprimorar a avaliação deste construto em pessoas que sofreram queimaduras, utilizando-se de instrumentos válidos e confiáveis.

$\mathrm{Na}$ literatura, há diversas definições de qualidade de vida e seus domínios. Considerando o contexto das pessoas com feridas, destacam-se a avaliação dos sintomas físicos, como dor, condições da queimadura, secreção e odor da lesão; da vida diária, como a pessoa gerencia sua vida e as questões sobre trabalho, exercícios físicos, atividades de lazer e diversão, dificuldades físicas e financeiras relacionadas à queimadura; da vida social e do bem estar psicológico, aspectos esses que se relacionam com os sentimentos de conviver com outras pessoas e do enfrentamento diante do trauma. Faz-se necessário também avaliar os aspectos que englobam o tratamento, tais como as questões relacionadas aos sentimentos do paciente em relação ao seu tratamento, a ajuda que outras pessoas fornecem e o tempo necessário por dia para cuidar da queimadura; e ainda, a satisfação global desse paciente com seu tratamento e sua nova aparência ${ }^{(7)}$.

Recentemente, resultados de uma revisão sistemática apontaram que os domínios mais afetados da QV e estado de saúde foram trabalho, sensibilidade da pele, dor, limitação física, função emocional ${ }^{(15)}$. No presente estudo, destaca-se que os participantes apresentaram melhores escores para os domínios referentes à saúde mental, como o Psicológico do WHOQOL-abreviado e, Afeto e Imagem corporal da BSHS-R. Corroborando com a literatura, nesta amostra, também se teve piores escores para os domínios Trabalho e Físico.
Em relação as características da amostra estudada no presente estudo, os resultados não se diferem de outros apresentados na realidade brasileira, com grande parte da amostra composta por homens, jovens, sendo o local de acidente prevalente o domiciliar, seguido do trabalho, e a escaldadura 0 agente causal das lesões ${ }^{(16-17)}$.

Destaca-se que em termos de relações interpessoais, avaliada pela escala BSHS-R, as mulheres apresentaram menores escores, sugerindo que elas percebem como pior sua QV neste aspecto. Em outro estudo, realizado com uma amostra pequena de mulheres, os autores discutem a possibilidade de mulheres terem mais dificuldades na recuperação ao longo do primeiro ano após a alta, dado que as mesmas apresentaram menos mudanças nos escores de QV, quando comparada aos homens ${ }^{(18)}$.

Conforme exposto acima, o tempo pós queimadura influencia na percepção de estado de saúde, no sentido de que com o passar do tratamento, as pessoas tendem a ter mais QV. Assim, neste estudo, encontrou-se que o tempo de tratamento foi associado com pior percepção de Afeto e imagem corporal, relacionamento interpessoal e melhor QV em habilidades para funções simples. Esses resultados sugerem que ao longo do tempo, as habilidades físicas podem retornar ao mais próximo do que era antes e que os aspectos relacionados à nova imagem corporal e os papeis sociais, inclusive dos relacionamentos podem estar prejudicados. Resultados estes auxiliam a direcionar intervenções para a reinserção social.

Relativo aos dados do trauma, a atual investigação observou maior números de casos de queimaduras em várias partes do corpo, com maior prevalência nos membros superiores e inferiores, corroborando com resultados de outros estudos ${ }^{(17-18)}$. Embora os participantes deste estudo tenham apresentado em média uma pequena área de superfície corporal queimada, com profundidade de espessura parcial, todos foram encaminhados e tratados em centro de tratamento especializado para essa população, o que garante um tratamento multidisciplinar, voltado para questões funcionais, estéticas e emocionais.

Os resultados deste estudo apontam que SCQ está associada negativamente com aspectos relacionados ao físico, tratamento, sensibilidade da pele, relações interpessoais e do estado de saúde geral. Na literatura, há resultados que apontam na mesma direção, principalmente em queimaduras mais extensas $(>9,5 \%)^{(19)}$. Ain$\mathrm{da}$, considerando os aspectos clínicos, observa-se que a grande maioria apresentou queimaduras em membros superiores e essa visibilidade das lesões e cicatrizes foi descrita em $80 \%$ da presenta amostra. Conforme apresentado por outros autores, queimaduras visíveis podem influenciar mudanças nos hábitos de se vestir, nos quais as pessoas tentam encobrir as áreas com sequelas e essa 
mudança no hábito foi associada a queimaduras mais extensas e também àquelas acometidas nos membros ${ }^{(20)}$

Embora este estudo apresente uma amostra de um único centro, os dados aqui encontrados corroboram com a literatura e reforçam a necessidade de se elaborar programas de reabilitação que englobe a individualidade e os múltiplos aspectos que influenciam a $\mathrm{QV}$ da pessoa que sofreu queimaduras.

A limitação do estudo está relacionada a coleta de dados, a qual foi realizada com o objetivo inicial de adaptação e validação de um instrumento de qualidade de vida e não especificamente para a análise na $\mathrm{QV}$ nessa população. Contudo, um dos testes estatísticos de validação no estudo primário utiliza-se de outro instrumento de $\mathrm{QV}$, o que permitiu a efetivação da pesquisa.

\section{CONCLUSÕES}

Este estudo demonstrou que há aspectos relacionados ao trauma, como a superfície corporal queimada, tempo de tratamento e ser do sexo feminino pode influenciar negativamente na percepção da qualidade de vida e estado de saúde de pessoas que sofreram queimaduras. Entretanto, o tempo de tratamento pode estar relacionado a uma melhora da percepção das habilidades simples. Recomenda-se que atenção especial seja dada aos aspectos físicos, relacionados ao trabalho, autoimagem e relacionamentos na reintegração social dessas pessoas na prática clínica e que as equipes multiprofissionais possam levar esses aspectos em considerações para o desenvolvimento de programas de reabilitação.

Neste sentido, outros estudos devem ser empreendidos avaliando os aspectos que interferem na qualidade de vida, como uma medida de avaliação de intervenções e tratamento de saúde. $O$ enfermeiro é o profissional indicado para o cuidado holístico, interdisciplinar, com um importante poder de conhecimento para a tomada de decisão para o melhor tratamento, fazendo a diferença na vida das pessoas.

\section{DECLARAÇÃO DE CONFLITO DE INTERESSE FINANCEIRO E/OU DE AFILIAÇÕES}

Não há. 


\section{REFERÊNCIAS}

I. Rocha JLFN, Canabrava PBE, Adorno J, Gondim MFN. Qualidade de vida dos pacientes com sequelas de queimaduras atendidos no ambulatório da unidade de queimados do Hospital Regional da Asa Norte. Rev Bras Queimaduras.2016;15(I):3-7. Disponível em: http://www.rbqueimaduras.com.br/details/286/pt-BR/

2. Spronk I, Bonsel GJ, Polinder S, van Baar ME, Janssen MF, Haagsma JA. Exploring the relation between the EQ-5D-5L pain/discomfort and pain and itching in a sample of burn patients. Health Qual Life Outcomes. 2020 May 19;/8(I):I44. Disponível em: https://www. ncbi.nlm.nih.gov/pmc/articles/PMC7236/21/

3. Gojowy D, Kauke M, Ohmann T, Homann HH, Mannil L. Early and late-recorded predictors of health-related quality of life of burn patients on long-term follow-up. Burns. 2019;45(6): | 300-I3 | 0 . Disponível em: https:// pubmed.ncbi.nlm.nih.gov/3 I I76508/

4. Civile VT, Finotti CS. Abordagem fisioterapêutica precoce em pacientes críticos queimados. Rev Bras Queimaduras.20 I2; I (2):85-88. Disponível em: http:// www.rbqueimaduras.com.br/details/ / 07/pt-BR/

5. World Health Organization. WHOQOL: Measuring quality of life. Acesso em 30 de julho de 2020. Disponível em: https:/www.who.int/healthinfo/survey/whoqol-qualityoflife/en/

6. de Assis SCJ, da Silva FMW, Albino SC, de Souza SF, Barreto FJ. Sentimentos vivenciados por mulheres vítimas de queimaduras: revisão integrativa. Revista Pesquisa fundam. Online. 20I5. dez. 7(supl.):56-64. Disponível em: https://dialnet.unirioja.es/servlet/articulo? codigo $=5618287$

7. Kaizer UAO.Propriedades psicométricas do "Freiburg Life QualityAssessment Wound" em queimados [dissertação mestrado]. Campinas:Universidade Estadual de Campinas, Faculdade de Enfermagem; 20I4. Disponível em: http://repositorio.unicamp.br/handle/REPOSIP/283873

8. Ferreira E, Dantas RAS, Rossi LA, Ciol MA. The cultural adaptation and validation of the Burn Specific Health Scale-Revised (BSHS-R):Version for Brazilian burn victims. Burns. 2008;34(7):994-I00I. Disponível em: https://pubmed.ncbi.nlm.nih.gov//8375073/

9. Blalock SJ, Bunker BJ, DeVellis RF. Measuring health status among survivors of burn injury: revisions of the Burn Specific Health Scale. J Trauma. 1994;36(4):508-I5.Disponível em: https://pubmed.ncbi.nlm.nih.gov/81587/2/

10. The Whoqol Group. Development of the World Health Organization WHOQOL-bref. Quality of Life Assesment 1998. PsycholMed. 1998;28:55 I-8. Disponível em: https://pubmed.ncbi.nlm.nih.gov/96267/2/

II.Fleck MPA, Louzada S, Xavier M, Chachamovich E, Vieira G, Santos L, et al. Aplicação da versão em português do instrumento abreviado de avaliação da qualidade de vida "WHOQOLBref". Rev saúde pública. 2000;34(2):I78-83. Disponível em: https:// www.scielo.br/scielo.php?script=sci_arttext\&pi$d=S 0034-89$ | $020000002000 / 2$

12. Cohen J. Statistical power analysis for the behavioral sciences. $2^{\mathrm{a}}$ ed. New Jersey: Lawrence Erlbaum Associates; 1988. The significance of a product moment rs. p.75- 108.

13. Costa MC, Rossi LA, Dantas RAS, Trigueiros LF. Imagem Corporal e Satisfação no Trabalho Entre Adulto em Reabilitação de Queimaduras. CogitareEnferm. 2010 Abr/Jun; I5(2):209-16. Disponível em: http://dx. doi.org/I0.5380/ce.vI 5i2. 17849

I4.Zapata DM, Estrada E. Calidad de vida relacionada com la salud de las personas afectadas por quemaduras depúes de la cicatrizacion, Medellin, Colombia. Biomédica. 2010; 30(4):492-500. Disponível em: https://revistabiomedica.org/index.php/biomedica/article/view/287

I5.Spronk I, Legemate C, Oen I, van Loey N, Polinder $\mathrm{S}$, van Baar M. Health related quality of life in adults after burn injuries: A systematic review. PLoSOne. 20 I8; I3(5):e0 197507. Published 20 I 8 May 24. Disponível em: https://pubmed.ncbi.nlm.nih.gov/297956/6/

16. Souza SA, Carmo SA, Costa RA, de Lima G, Leonardo RNC. Características clínico-epidemiológicas de pacientes internados em um hospital de referência em queimaduras na Amazônia brasileira. Rev Bras Queimaduras. 2019;18(2):102-I06. Disponível em: http:// www.rbqueimaduras.com.br/details/464/pt-BR/

17. Malta DC et al. Perfil dos casos de queimadura atendidos em serviços hospitalares de urgência e emergência nas capitais brasileiras em 2017. Rev. bras. epidemiol. 2017;23(suppl I)e200005. Disponível em: https://www.scielo.br/scielo.php?script=sci_arttext\&pid=SI 4I5-790X2020000200403\&tlng=pt

18. Echevarría-Guanilo ME, Gonçalves N, Farina JA, Rossi LA.Avaliação da qualidade de vida relacionada à saúde no primeiro ano após a queimadura. Rev Esc Anna Nery 2016;20(I):I55-I66. Disponível em: https://doi. org/I0.5935/I4|4-8I45.20I6002I.

19. Kishawi D, Wozniak AW, Mosier MJ.TBSA and length of stay impact quality of life following burn injury. Burns. 2020 May;46(3):616-620. Disponível em: https:// doi.org/I0.1016/j.burns.2019.09.007

20. Echevarría-Guanilo ME, Martins CL, Cantarelli KJ, Gonçalves N, Rossi LA. Visibilidade das cicatrizes de queimaduras percebida pelos pacientes durante 0 primeiro ano de reabilitação. RevBras Queimaduras20I2;II(3):I20-I24. Disponível em: http://www. rbqueimaduras.com.br/details/l/6/pt-BR/

Recebido: 2020-09-23

Aceito: $2020-1$ I-30 